\title{
Contractile profile of esophageal and gastric fundus strips in experimental doxorubicin-induced esophageal atresia
}

\author{
F.A. Capeto ${ }^{1}$, F.J.B. Lima ${ }^{2}$, W. Okoba ${ }^{2}$, F.L. Ramos ${ }^{1}$, T.F.A. Messias ${ }^{1}$, G.A. Rigonatto ${ }^{1}$, \\ L. Sbragia ${ }^{3}$, P.J.C. Magalhães ${ }^{2}$ and A.A. Melo-Filho ${ }^{1}$ \\ ${ }^{1}$ Departamento de Cirurgia, Faculdade de Medicina, Universidade Federal do Ceará, Fortaleza, CE, Brasil \\ ${ }^{2}$ Departamento de Fisiologia e Farmacologia, Faculdade de Medicina, Universidade Federal do Ceará, Fortaleza, CE, Brasil \\ ${ }^{3}$ Departamento de Cirurgia e Anatomia, Faculdade de Medicina de Ribeirão Preto, \\ Universidade de São Paulo, Ribeirão Preto, SP, Brasil
}

\begin{abstract}
Esophageal atresia (EA) is characterized by esophageal and gastric motility changes secondary to developmental and postsurgical damage. This study evaluated the in vitro contractile profile of the distal esophagus and gastric fundus in an experimental model of EA induced by doxorubicin (DOXO). Wistar pregnant rats received DOXO $2.2 \mathrm{mg} / \mathrm{kg}$ on the 8th and 9th gestational days. On day 21.5 , fetuses were collected, sacrificed, and divided into groups: control, DOXO without EA (DOXO-EA), and DOXO with EA (DOXO + EA). Strips from the distal esophagus and gastric fundus were mounted on a wire myograph and isolated organ-bath system, respectively, and subjected to increasing concentrations of carbamylcholine chloride (carbachol, CCh). The isolated esophagus was also stimulated with increasing concentrations of $\mathrm{KCl}$. In esophagus, the concentration-effect curves were reduced in response to $\mathrm{CCh}$ in the $\mathrm{DOXO}+\mathrm{EA}$ and DOXO-EA groups compared to the control group $(\mathrm{P}<0.05)$. The maximum effect values $\left(\mathrm{E}_{\max }\right)$ for $\mathrm{DOXO}+\mathrm{EA}$ and DOXO-EA were significantly lower than control $(P<0.05)$, but the half-maximal effective concentration $\left(E_{50}\right)$ values were not significantly different when the three groups were compared $(P>0.05)$. In response to $\mathrm{KCl}$, the distal esophagus samples in the three groups were not statistically different with regard to $E_{\max }$ or $E C_{50}$ values $(P>0.05)$. No significant difference was noted for $E C_{50}$ or $E_{\max }$ values in fundic strips stimulated with $\mathrm{CCh}(\mathrm{P}>0.05)$. In conclusion, exposure of dams to DOXO during gestation inhibited the contractile behavior of esophageal strips from offspring in response to $\mathrm{CCh}$ but not $\mathrm{KCl}$, regardless of EA induction. The gastric fundus of DOXO-exposed offspring did not have altered contractile responsiveness to cholinergic stimulation.
\end{abstract}

Key words: Esophageal atresia; Doxorubicin; Experimental; Esophageal contractility

\section{Introduction}

Esophageal atresia (EA) is a primary structural anomaly that results from incomplete esophagealtracheal septation during intrauterine development, with associated varied lesions in the distal esophagus and stomach during its development or after surgical correction. Dysmotility-associated symptoms such as gastroesophageal reflux (GER) and dysphagia are frequent in the affected population, persisting through adulthood and thus negatively impacting quality of life (1-3).

Experimental and clinical studies have previously shown the existence of altered intrinsic innervation in $E A$ $(4,5)$. However, data on esophageal and gastric motility profiles are scarce. Furthermore, the few available published clinical studies on patients with EA with tracheoesophageal fistula (TEF) have failed to show the contractile nature of the esophagus after surgical correction, probably due to technical limitations (6-10). Therefore, it is difficult to separate postsurgical and embryological alterations. Experimentally induced EA has been useful for identifying and understanding esophageal and gastric motility changes attributable to development. The teratogenesis of doxorubicin (DOXO) is useful for this purpose $(11,12)$.

The main objective of this work was to study in vitro the gastric and esophageal contractility in an experimental model of EA induced by DOXO in rats to determine if it is a useful model for dysmotility-associated symptoms.

\section{Material and Methods}

\section{Animals}

The experimental protocol was approved by the Ethics and Research on Animals Committee (CEPA \#27/2011)

Correspondence: A.A. Melo-Filho: <aamelofilho@gmail.com>. 
at the Universidade Federal do Ceará. Female albino Wistar rats $(\sim 250 \mathrm{~g})$ were obtained from the Central Vivarium, Universidade Federal do Ceará. They were maintained under an acclimatized day/night ambience, with free access to food and water. Under this condition, females were mated with males and natural reproduction was allowed, with verification carried out on the subsequent morning. Rats that showed a positive sperm-test on vaginal lavage were considered pregnant, with the date determined as day 0 (term $=22$ days). Pregnant rats were separated into single cages with the same conditions as prior to pregnancy.

\section{Experimental procedure}

The technique used for the induction of EA was described by França et al. (13). Doxorubicin chloride $(2.2 \mathrm{mg} / \mathrm{kg}$ ) was administered (intra-peritoneal injections using a solution of $2 \mathrm{mg} / \mathrm{mL}$ DOXO in saline) once daily on days 8 and 9 of pregnancy $(n=26)$. Another group of pregnant rats $(n=13)$ received only the saline vehicle.

\section{Data collection}

On day 21.5, pregnant rats were anesthetized with an intramuscular injection of $1.25 \mathrm{~mL} / \mathrm{kg}$ ketamine $(50 \mathrm{mg} /$ $\mathrm{mL}$ ) and $0.25 \mathrm{~mL} / \mathrm{kg}$ xylazine $(10 \mathrm{mg} / \mathrm{mL})$ and subsequently subjected to caesarian surgery to remove the fetuses. The bicornuate uterus was exposed, and the respective fetuses were identified numerically from the most proximal to the cervix to the most distal. Afterward, the fetuses were weighed before being sacrificed by occipital puncture and subjected to a cervico-thoraco-laparotomy using a surgical magnifying lens (Magnifier Lamp, Model TL1092, TÜV Rheinland ${ }^{\mathbb{R}}$, Germany) to detect EA and associated TEF. Thereafter, the distal esophageal tissue and gastric fundic strips were obtained for in vitro contractile studies. Tissues were obtained from animals divided into three groups: control (rats that received only vehicle during pregnancy), DOXO-EA (dams injected with DOXO during pregnancy, but the fetus did not develop EA) and DOXO +EA (dams injected with DOXO during pregnancy and the fetus developed EA).

\section{In vitro esophageal contractility protocols}

The isolated esophagus was cut transversally to produce ring-like segments (approximately 2-mm length) that were mounted horizontally onto a wire myograph (610M-DMT; DMT, Aarhus, Denmark). Mechanical tension was applied to each segment by two tungsten wires $(40 \mu \mathrm{m})$ that were extended parallel through the esophageal lumen. One wire was connected to a highly sensitive force transducer to record isometric contractions, while the other was connected to a micrometer that allowed basal tension adjustments (set at $8 \mathrm{mN}$ ). The tissue was constantly bathed with a physiological Tyrode solution $(136.0 \mathrm{mM} \mathrm{NaCl}, 5.0 \mathrm{mM} \mathrm{KCl}, 0.98 \mathrm{mM}$ $\mathrm{MgCl}_{2}, 2.0 \mathrm{mM} \mathrm{CaCl}, 0.36 \mathrm{mM} \mathrm{NaH} \mathrm{PO}_{4}, 11.9 \mathrm{mM}$ $\mathrm{NaHCO}_{3}$, and $5.5 \mathrm{mM}$ glucose) at $37^{\circ} \mathrm{C}$ that was continually bubbled with $5 \% \mathrm{CO}_{2}$ and $95 \% \mathrm{O}_{2}$, and the $\mathrm{pH}$ was adjusted to 7.4. A basal period of $1.5 \mathrm{~h}$ was established before initiating chemical stimulation. During this period, the perfusing liquid was changed after every $15 \mathrm{~min}$ and the basal tension was monitored. Consequently, a concentration-effect curve (CEC) was obtained after cumulatively pipetting several concentrations of carbamylcholine chloride (carbachol, CCh, $0.01-100 \mu \mathrm{M})$ or $\mathrm{KCl}(10-100 \mathrm{mM})$. The maximum effect $\left(E_{\max }\right)$ of a CEC was determined when a given concentration of the contractile agent did not elicit a contraction higher than that provoked by the preceding concentration. The halfmaximal effective concentration values $\left(E_{50}\right)$ of contractile agents were derived for each CEC.

\section{In vitro gastric contractility protocol}

Fundic tissues were obtained as ring-like strips from the fetal stomach cut in the coronal plane. Two nonextensible cotton purse strings were used: one to maintain the tissue fixed on a metal haste, and the other to maintain the ring on a highly sensitive force isometric transducer (ML870B60/CV, AD Instruments, New South Wales, Australia) that was subsequently connected to a digital data acquisition system (PowerLab ${ }^{\mathrm{TM}}$ 8/30, AD Instruments). Each tissue bath contained $5 \mathrm{~mL}$ of Tyrode solution ( $\mathrm{pH} 7.4$ ), heated to $37^{\circ} \mathrm{C}$ and constantly oxygenated with $5 \% \mathrm{CO}_{2}$ in $95 \% \mathrm{O}_{2}$. Each successful set up was calibrated to a basal tension of $1 \mathrm{~g}$ over $1 \mathrm{~h}$. During this period, the perfusing fluid was changed every $15 \mathrm{~min}$, and the basal tension was adjusted. To test the viability of each tissue set-up after the basal period, two successful contractions were induced by adding $80 \mathrm{mM} \mathrm{KCl}$ into the bath. Thereafter, each strip was stimulated with increasing concentrations of $\mathrm{CCh}(0.01-300 \mu \mathrm{M})$ to obtain a respective CEC. At the end of each protocol, each isolate was weighed, and the contractile force was expressed as its relationship to the respective tissue mass (g tension/ $\mathrm{mg}$ of tissue). The respective $\mathrm{E}_{\max }$ and $\mathrm{EC}_{50}$ values were derived at the end of the experiment.

\section{Statistical analysis}

Data for esophageal and gastric contractility are reported as means $\pm S E$, with $n$ indicating the number of experiments. Statistical analysis was determined with two-way variance analysis (ANOVA), followed by StudentNewman-Keuls tests when results were significant. The respective $\mathrm{EC}_{50}$ values were obtained by semi-logarithmic interpolation and are reported as the geometric mean [95\% confidential interval] and the number of experiments. We compared the respective $E_{50}$ values using Mann-Whitney $U$-tests. The $E_{\max }$ values were determined after obtaining the maximum contractile response. $\mathrm{P}<0.05$ was considered statistically significant.

\section{Results}

Twenty-six DOXO-exposed pregnant rats produced 
164 fetuses, with 17 (10.36\%) showing macroscopic EA with distal TEF.

\section{Evaluation of the contractile behavior of distal esophageal strips in response to $\mathrm{CCh}$}

In isolated strips from the control group, cumulative concentrations of CCh $(0.01-100 \mu \mathrm{M})$ produced a CEC with an $E_{\max }$ of $5.97 \pm 0.58 \mathrm{mN}(n=11)$ that was obtained at $100 \mu \mathrm{M}$ (Figure 1). It was significantly higher than that obtained from isolated tissues from animals exposed to DOXO $(\mathrm{P}<0.05$, two way ANOVA, Student-NewmanKeuls; Table 1). The decreased $E_{\max }$ value under cholinergic stimuli was independent of the presence of $E A ; E_{\max }$ values observed for both the DOXO-EA and $\mathrm{DOXO}+\mathrm{EA}$ groups were significantly lower than that measured in the control group, but they were not significantly different from each other $(P>0.05$, ANOVA; Table 1). Regarding the $\mathrm{EC}_{50}$ values for $\mathrm{CCh}$-induced contractions, there was no significant difference among the three groups ( $P>0.05$, Mann-Whitney U-test; Table 1).

\section{Evaluation of the contractile behavior of distal esophageal strips in response to $\mathrm{KCl}$}

After exposure to increasing concentrations of $\mathrm{KCl}$ (10-100 mM), esophageal strips in the control group produced a CEC with an $E_{\max }$ of $1.31 \pm 0.14 \mathrm{mN}(n=5)$, which was not significantly different from the values in groups pre-treated with DOXO $(P>0.05$, two-way ANOVA; Table 1 and Figure 2).

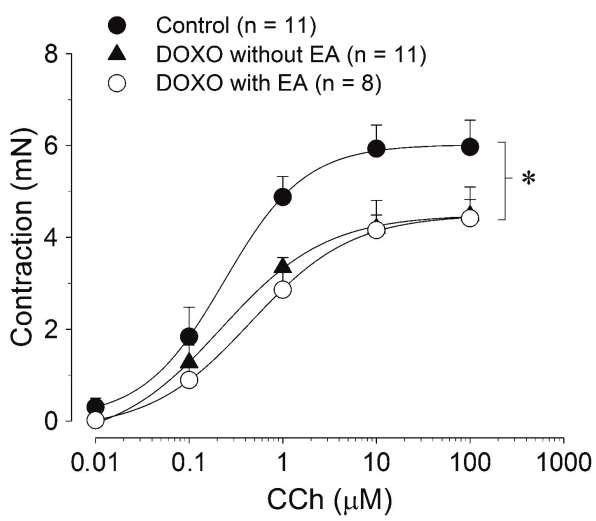

Figure 1. Comparison of the effect of carbachol (CCh) on the distal esophagus of fetus with esophageal atresia (EA). The graph shows the mean tension of the distal esophageal strips in response to increasing concentrations of CCh $(0.01-100 \mu \mathrm{M})$. The tension was verified considering difference in peak and the amplitude expressed in $\mathrm{mN}$. Data are reported as means $\pm \mathrm{SE}$. DOXO: doxorubicin. ${ }^{*} \mathrm{P}<0.05$, treated groups compared to controls (two-way ANOVA, followed by a Student-NewmanKeuls test).

\section{Evaluation of the contractile behavior of fundic gastric strips in response to $\mathrm{CCh}$}

The mean weights of fundic strips were $3.80 \pm 0.33$, $4.10 \pm 0.35$, and $3.77 \pm 0.44 \mathrm{mg}$, for the control, DOXO$E A$, and DOXO + EA groups, respectively. These values were not significantly different $(P>0.05$, one-way ANOVA). Exposure of fundic strips from the control group to increasing concentrations of CCh $(0.01-300 \mu \mathrm{M})$ resulted in contractile responses with an $E_{\max }$ of $0.084 \pm 0.016 \mathrm{~g} / \mathrm{mg}$ of tissue $(n=7)$ at a concentration of $100 \mu \mathrm{M}$ (Figure 3). However, the respective values in the DOXO-EA and DOXO + EA groups were not significantly different ( $P>0.05$, two-way ANOVA; Table 2). Similarly, the respective $\mathrm{EC}_{50}$ values were not statistically different between the three groups $(\mathrm{P}>0.05$, Mann-Whitney U-test; Table 2).

\section{Discussion}

It is well known that even after surgical correction, babies born with EA continue to experience functional repercussions. The most common complication is gastroesophageal reflux disease (GERD), with varied symptom intensity. GERD-related symptoms seem to stem from the initial EA-induced lesions on two primordial protective barriers to reflexive episodes: the mechanical and physiological entity of the esophageal-gastric junction and the esophagus clearance mechanism (3,6-8). Particularly, the lack of distal esophageal contractions is significantly correlated with the development of GERD (14). This may be demonstrated by a conventional or a high-resolution postsurgical esophageal manometry that may reveal alterations including absence of or uncoordinated peristaltic waves, simultaneous contractions, low amplitude contractions, and high base-line pressure $(9,10)$.

It is thought that surgical intervention of the esophagus, even for therapeutic purposes, is capable of negatively impacting esophageal motility, probably due to vagal denervation whether by operational tractionmechanisms or cranial dislocation of the esophagogastric junction due to surgery (3). However, these mechanisms do not negate the fact that congenital alterations affect motility mechanisms prior to diagnosis and therapy. Clinical presurgical evaluation of neonates with EA routinely reveals esophageal dysmotility (15). Moreover, esophageal motility is also severely affected in children with isolated TEF prior to surgery (16).

There are three types of animal models used to study EA: teratogen-induced, surgically created, and knockout models. Among surgical models, the chick embryo does not reliably mimic EA and TEF. Another surgical model is producing esophageal lesions in puppies to study postnatal surgical correction techniques. On the other hand, knockout mice are useful for studying genetic influences. The DOXO rodent model is the most useful for investigating the morphology and faulty organogenesis associated 
Table 1. Values of $\mathrm{E}_{\max }$ for $\mathrm{CCh}$ and $\mathrm{KCl}$ and values of $\mathrm{EC}_{50}$ for $\mathrm{CCh}$ in segments of the distal esophagus.

\begin{tabular}{lccc}
\hline & Control & DOXO-EA & DOXO +EA \\
\hline $\mathrm{CCh}$ & $(\mathrm{n}=11)$ & $(\mathrm{n}=11)$ & $(\mathrm{n}=8)$ \\
$\mathrm{E}_{\max }(\mathrm{mN})$ & $5.97 \pm 0.58^{*}$ & $4.48 \pm 0.34$ & $4.42 \pm 0.68$ \\
$\mathrm{EC}_{50}(\mathrm{nM})$ & $190[96-379]$ & $228[125-418]$ & $439[206-936]$ \\
$\mathrm{KCl}$ & $(\mathrm{n}=5)$ & $(\mathrm{n}=7)$ & $(\mathrm{n}=7)$ \\
$\mathrm{E}_{\max }(\mathrm{mN})$ & $1.31 \pm 0.14$ & $1.27 \pm 0.42$ & $1.21 \pm 0.20$ \\
\hline
\end{tabular}

Data are reported as means \pm SE or geometric mean $[95 \% \mathrm{Cl}]$. DOXO-EA: doxorubicin without esophageal atresia; DOXO +EA: doxorubicin with esophageal atresia; CCh: carbachol; $E_{\text {max: }}$ maximum effect values in $\mathrm{mN}$; $\mathrm{EC}_{50}$ : half-maximal effective concentration. ${ }^{*} \mathrm{P}<0.05$ (two-way ANOVA, StudentNewman-Keuls test).

with gene-expression regulation in EA. Even though the anomaly is secondarily induced by an external agent (a teratogenic drug) and its incidence in treated fetuses is variable (which necessitates a higher number of animals), the model is clinically relevant with regard to phenotypic similarity. In addition, the rat model provides fetal samples that can be macroscopically evaluated $(13,17,18)$.

With the intent of clarifying the primary etiology of this anomaly, the DOXO experimental model has shown that the length of the intra-abdominal esophagus is shorter, but the size of the esophageal hiatus is larger, than in controls (19). Using the same model, Qi et al. (20) found fewer extrinsic innervations with respect to both of the recurrent laryngeal nerves, deviation of the left vagus from its normal course to form a single nerve trunk on the right side of the esophagus, and relatively few branches from the single vagal nerve trunk.

Diverse studies have correlated primary motility dysfunction with intrinsic innervation alteration, including

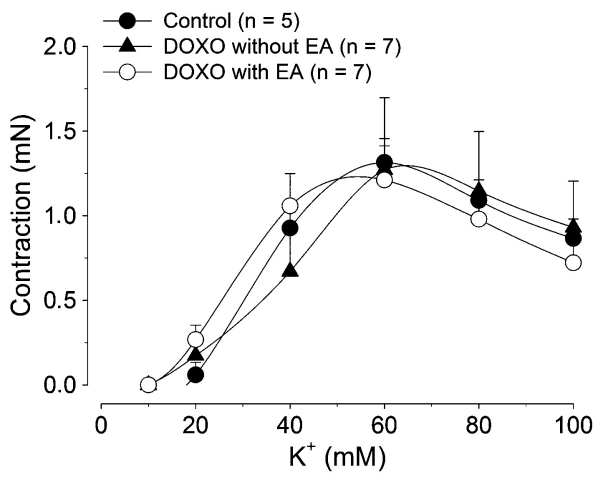

Figure 2. Comparison of the effect of $\mathrm{KCl}$ on the distal esophagus of fetus with esophageal atresia (EA). The graph shows the mean tension of the distal esophageal strips in response to increasing concentrations of $\mathrm{KCl}(10-100 \mathrm{mM})$. The tension was verified considering difference in peak and the amplitude expressed in $\mathrm{mN}$. Data are reported as means $\pm \mathrm{SE}$. DOXO: doxorubicin. There were no statistical differences in contractile activity in response to $\mathrm{KCl}$ comparing treated groups to controls $(P>0.05$, two-way ANOVA, followed by a StudentNewman-Keuls test). intrinsic nerve complex size and limited distribution and immaturity of nerve tissue at the distal esophagus tissue, especially where there is an association with TEF $(4,5)$. Midrio et al. (21) demonstrated that in neonates with EA/ TEF, there is a lower density of the interstitial cells of Cajal at the tip of the trachea.

With the goal of elucidating the pathophysiology of motor dysfunctions, in vitro experimental studies may provide a way to explain this phenomenon at the tissue level. However, few studies that employed the methodology of the present study are available, in particular exploiting DOXO teratogenicity and analyzing the contractility of the esophagus and stomach of rat fetuses $(11,12)$. In the studies published by Tugay et al. $(11,12)$, the effects of specific agonists such as CCh, serotonin, and isoproterenol were evaluated, and the effects of unspecific compounds such as $\mathrm{KCl}$ and papaverine on smooth muscle cells were also addressed. No significant differences were noted in relation to CCh-elicited contractile

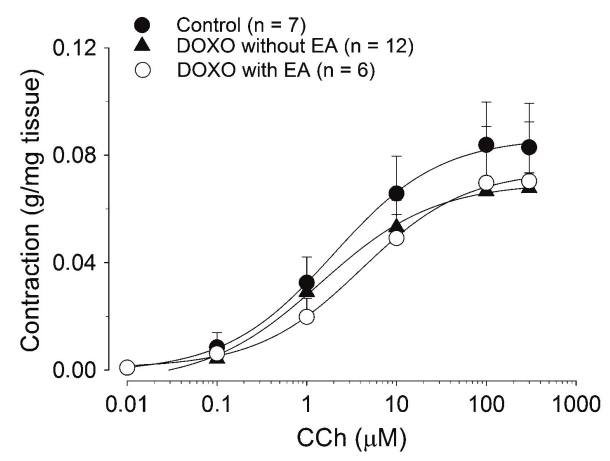

Figure 3. Comparison on the effect of carbachol (CCh) on the gastric fundic strips of fetus with EA. The graph shows the mean tension of the gastric fundic strips in response to increasing concentrations of CCh $(0.01-300 \mu \mathrm{M})$. The tension was verified considering difference in peak and the amplitude expressed as $\mathrm{g}$ (force)/mg (tissue mass). Data are reported as means $\pm S E$. DOXO: doxorubicin. There were no statistical differences in contractile activity in response to CCh comparing treated groups to controls $(P>0.05$, two-way ANOVA, followed by a StudentNewman-Keuls test). 
Table 2. Values of $E_{\max }$ and the $\mathrm{EC}_{50}$ for carbachol $(\mu \mathrm{M})$ in gastric fundic strips.

\begin{tabular}{cccc}
\hline & Control & DOXO-EA & DOXO +EA \\
\hline CCh & $(n=7)$ & $(n=12)$ & $(n=6)$ \\
$E_{\max }$ & $0.084 \pm 0.016$ & $0.068 \pm 0.006$ & $0.070 \pm 0.022$ \\
EC $_{50}$ & $2.17[1.03-4.58]$ & $1.47[0.83-2.61]$ & $3.26[1.90-5.60]$ \\
\hline
\end{tabular}

Data are reported as means \pm SE or geometric mean $[95 \% \mathrm{Cl}]$. DOXO-EA: doxorubicin without esophageal atresia; DOXO +EA: doxorubicin with esophageal atresia; $\mathrm{CCh}$ : carbachol; $\mathrm{E}_{\max }$ : maximum effect values in $\mathrm{g} / \mathrm{mg}$ tissue mass; $\mathrm{EC}_{50}$ : half-maximal effective concentration.

response in lower esophageal strips from fetus pretreated with DOXO, despite the fact that a significantly lower capacity of muscle relaxation in response to serotonin was noted in this tissue (11).

In the present study, the muscarinic agonist CCh elicited concentration-dependent contraction in esophageal strips, even in tissues from the DOXO group. However, esophageal strips of animals treated with DOXO demonstrated a smaller maximum force of contraction than the control group. The decrease in the pharmacological capacity of CCh to produce a contractile response does not appear to be due to EA development. Notably, both groups subjected to DOXO (with or without EA) showed reduced contractility in response to cholinergic stimulus. This difference may be due to treatment with DOXO, and it was not extended to gastric tissues because fundic rings reacted similarly to CCh-induced stimulation, as discussed below.

It is worth noting that Qi et al. (22) employed a similar model and detected reductions in the density and distribution of the myoenteric plexus, specifically regarding vasoactive intestinal peptide- (VIP-) and substance P-producing neurons in DOXO pretreated animals. Such findings revealed the deleterious effects of DOXO in the intrinsic innervations of the esophagus, which are able to change the mechanisms of signal transduction in affected tissues independently of morphological alterations due to its teratogenicity. Notwithstanding, it is important to mention that while Tugay et al. (11) utilized whole-mount preparations that allowed contractile recordings of the longitudinal smooth muscle layer of the lower esophagus, we employed esophageal strips arranged to record circular smooth muscle contractions. Thus, it is possible that the decreased responsiveness of the distal esophagus to cholinergic stimulus may be restricted to functional changes in the circular smooth muscle layer.

In our studies, the $\mathrm{KCl}$ concentration-effect curves of the three groups were not significantly different in esophageal strips. Unlike CCh, which causes contractions mainly after intracellular release of $\mathrm{Ca}^{2+}$ stores and subsequent influx from the extracellular medium, $\mathrm{KCl}$-induced contraction results from its depolarizing properties on the cell membrane that stimulate the opening of voltage-operated $\mathrm{Ca}^{2+}$ (VOC) channels and $\mathrm{Ca}^{2+}$ influx into the cytosol (23). So, it is reasonable to hypothesize that pretreatment with DOXO did not interfere with the capacity of the esophageal cells to recruit VOC channels in inducing motility. These findings corroborate those of Tugay et al. (11).

Few published studies have analyzed gastric motility in neonates with EA. Some have identified antral hypomotility and reduced gastric emptying by utilizing electrogastrography, gastric cintilography, and manometry, but such events were not directly related to GERD episodes $(24,25)$. This renders the etiologies of these alterations and their roles in gastric motility obscure. However, it is debatable whether the intrinsic nervous system of the stomach is also affected in cases of embryologic EA or if such alterations are consequential to surgical interventions that involve gastric tractions. For instance, Zhou et al. (19) found that the EA fetuses of DOXO-treated rats possessed smaller stomachs than controls.

With regard to CCh stimulation, we did not find any significant difference in contractility profiles when comparing the fundic strip responses of the three groups. However, Tugay et al. (12) demonstrated that stomach strips of rat fetuses with EA due to DOXO exhibited significant differences in contractility. While the contractile response to CCh was increased, the contraction induced by serotonin was blunted. This difference may be due to methodological variability between the two studies. Our isolated fundic preparations were mounted after obtaining continuous circular muscle strips in the form of tissue rings. In contrast, Tugay et al. (12) adopted the method suggested by Vane (26) and registered longitudinal contractions of the gastric fundus. This difference raises the issue that in the gastric fundus, contrary to what is seen in the distal esophagus, the longitudinal layer is more responsive to cholinergic stimulation (12).

The DOXO treatment regimen was based on a previous protocol that induced EA in $91 \%$ of fetuses (13). The two studies published by Tugay et al. $(11,12)$ used a very similar dose (2 $\mathrm{mg} / \mathrm{kg}$ for the same two gestational days) and only reached rates of $42.85 \%$ and $57.69 \%$ of fetuses with EA. Notably, our rate was just $10.36 \%$. It is possible that the use of different rat strains may have contributed to variations among the studies. While the present approach was developed based on Wistar rats, other studies (11-13) utilized Sprague Dawley rats. Although the teratogenic 
effect of DOXO appeared less toxic, this dose was sufficient to induce EA and inhibit the in vitro contractile behavior of esophageal strips in response to CCh. Despite this, we acknowledge that more studies are needed to identify which factors contribute to functional alterations. Correlations of in vitro results with detailed histological evaluations of the esophageal and gastric tissues may improve our understanding about motility in EA.

\section{References}

1. Koivusalo A, Pakarinen MP, Rintala RJ. The cumulative incidence of significant gastrooesophageal reflux in patients with oesophageal atresia with a distal fistula - a systematic clinical, pH-metric, and endoscopic follow-up study. J Pediatr Surg 2007; 42: 370-374, doi: 10.1016/j.jpedsurg.20 06.10 .010 .

2. Ijsselstijn $H$, van Beelen NW, Wijnen RM. Esophageal atresia: long-term morbidities in adolescence and adulthood. Dis Esophagus 2013; 26: 417-421, doi: 10.1111/dote.12059.

3. Tovar JA, Fragoso AC. Anti-reflux surgery for patients with esophageal atresia. Dis Esophagus 2013; 26: 401-404, doi: 10.1111/dote.12063.

4. Boleken M, Demirbilek S, Kirimiloglu H, Kanmaz T, Yucesan $\mathrm{S}$, Celbis $\mathrm{O}$, et al. Reduced neuronal innervation in the distal end of the proximal esophageal atretic segment in cases of esophageal atresia with distal tracheoesophageal fistula. World J Surg 2007; 31: 1512-1517, doi: 10.1007/s00268-00 7-9070-y.

5. Cheng W, Bishop AE, Spitz L, Polak JM. Abnormal enteric nerve morphology in atretic esophagus of fetal rats with adriamycin-induced esophageal atresia. Pediatr Surg Int 1999; 15: 8-10, doi: 10.1007/s003830050500.

6. Frohlich T, Otto S, Weber P, Pilic D, Schmidt-Choudhury A, Wenzl TG, et al. Combined esophageal multichannel intraluminal impedance and $\mathrm{pH}$ monitoring after repair of esophageal atresia. J Pediatr Gastroenterol Nutr 2008; 47: 443-449, doi: 10.1097/MPG.0b013e3181638ca2.

7. Catalano P, Di Pace MR, Caruso AM, Casuccio A, De Grazia E. Gastroesophageal reflux in young children treated for esophageal atresia: evaluation with $\mathrm{pH}$-multichannel intraluminal impedance. J Pediatr Gastroenterol Nutr 2011; 52: 686-690, doi: 10.1097/MPG.0b013e318202a3e5.

8. Di Pace MR, Caruso AM, Catalano P, Casuccio A, Cimador M, De Grazia E. Evaluation of esophageal motility and reflux in children treated for esophageal atresia with the use of combined multichannel intraluminal impedance and $\mathrm{pH}$ monitoring. J Pediatr Surg 2011; 46: 443-451, doi: 10.1016/j.jpedsurg.2010.08.012.

9. Aspirot A, Faure C. Esophageal dysmotility: characterization and pathophysiology. Dis Esophagus 2013; 26: 405409, doi: $10.1111 /$ dote.12058.

10. Lemoine $C$, Aspirot A, Le Henaff $G$, Piloquet $H$, Levesque D, Faure C. Characterization of esophageal motility following esophageal atresia repair using high-resolution esophageal manometry. J Pediatr Gastroenterol Nutr 2013; 56: 609614, doi: 10.1097/MPG.0b013e3182868773.

11. Tugay M, Yildiz F, Utkan T, Ulak G, Gacar N, Erden F. Impaired esophageal reactivity in adriamycin-induced rat esophageal atresia: an in vitro study. J Pediatr Surg 2001;

\section{Acknowledgments}

The authors would like to thank Armênio A. Santos and Ronaldo A. Ribeiro for their valuable and constructive suggestions regarding this research. This study was supported by CNPq (\#2011/163018-1 and \#2009/ 479853-6).

36: 1569-1573, doi: 10.1053/jpsu.2001.27051.

12. Tugay M, Yildiz F, Utkan T, Sarioglu Y, Gacar N. Gastric smooth muscle contractility changes in the esophageal atresia rat model: an in vitro study. J Pediatr Surg 2003; 38: 1366-1370, doi: 10.1016/S0022-3468(03)00397-X.

13. França WM, Goncalves A, Moraes SG, Pereira LA, Sbragia L. Esophageal atresia and other visceral anomalies in a modified Adriamycin rat model and their correlations with amniotic fluid volume variations. Pediatr Surg Int 2004; 20: 602-608, doi: 10.1007/s00383-004-1240-5.

14. Kawahara H, Kubota A, Hasegawa T, Okuyama H, Ueno T, Watanabe T, et al. Lack of distal esophageal contractions is a key determinant of gastroesophageal reflux disease after repair of esophageal atresia. J Pediatr Surg 2007; 42: 20172021, doi: 10.1016/j.jpedsurg.2007.08.023.

15. Romeo G, Zuccarello B, Proietto F, Romeo C. Disorders of the esophageal motor activity in atresia of the esophagus. $J$ Pediatr Surg 1987; 22: 120-124, doi: 10.1016/S00223468(87)80425-6.

16. Vali K, Aspirot A, Morris M, Lemoine C, Giguere L, Faure C. Evidence of primary esophageal dysmotility in children with pure tracheo-esophageal fistula. Neurogastroenterol Motil 2011; 23: S40-S41.

17. Mortell A, Montedonico S, Puri P. Animal models in pediatric surgery. Pediatr Surg Int 2006; 22: 111-128, doi: 10.1007/ s00383-005-1593-4.

18. McLaughlin D, Hajduk P, Murphy P, Puri P. AdriamycinInduced models of VACTERL association. Mol Syndromol 2013; 4: 46-62.

19. Zhou B, Hutson JM, Myers NA. Investigation of the intraabdominal oesophagus and hiatus in fetal rats with oesophageal atresia and tracheo-oesophageal fistula. Pediatr Surg Int 2001; 17: 97-100, doi: 10.1007/s003830000456.

20. Qi BQ, Merei J, Farmer P, Hasthorpe S, Myers NA, Beasley SW, et al. The vagus and recurrent laryngeal nerves in the rodent experimental model of esophageal atresia. $J$ Pediatr Surg 1997; 32: 1580-1586, doi: 10.1016/S0022-3468(97) 90457-7.

21. Midrio P, Alaggio R, Strojna A, Gamba P, Giacomelli L, Pizzi $\mathrm{S}$, et al. Reduction of interstitial cells of Cajal in esophageal atresia. J Pediatr Gastroenterol Nutr 2010; 51: 610-617, doi: 10.1097/MPG.0b013e3181dd9d40.

22. Qi $B Q$, Uemura S, Farmer $P$, Myers NA, Hutson JM. Intrinsic innervation of the oesophagus in fetal rats with oesophageal atresia. Pediatr Surg Int 1999; 15: 2-7, doi: 10.1007/s003830050499.

23. Ribeiro-Filho HV, Brito TS, Lima FJ, Pinho JP, Sousa DF Silva MT, et al. Talking about bioelectrical potentials using rings of the mesenteric artery without glass micropipettes. 
Adv Physiol Educ 2012; 36: 336-344, doi: 10.1152/ advan.00050.2012

24. Romeo C, Bonanno N, Baldari S, Centorrino A, Scalfari G, Antonuccio $\mathrm{P}$, et al. Gastric motility disorders in patients operated on for esophageal atresia and tracheoesophageal fistula: long-term evaluation. J Pediatr Surg 2000; 35: 740744, doi: 10.1053/jpsu.2000.6048.
25. Bokay J, Kis E, Verebely $\mathrm{T}$. Myoelectrical activity of the stomach after surgical correction of esophageal atresia. $J$ Pediatr Surg 2005; 40: 1732-1736, doi: 10.1016/j.jpedsurg. 2005.07.031.

26. Vane JR. A sensitive method for the assay of 5-hydroxytryptamine. Br J Pharmacol Chemother 1957; 12: 344-349, doi: 10.1111/j.1476-5381.1957.tb00146.x. 